\title{
Wavelets in Medical Image Processing On Hip Arthroplasty and De-Noising, Segmentation
}

\author{
Himadri Nath Moulick ${ }^{1}$, Moumita Ghosh ${ }^{2}$ \\ ${ }^{1}$ CSE, Aryabhatta Institute of Engg \& Management, Durgapur, PIN-713148, India \\ ${ }^{2}$ CSE,University Institute Of Technology,(The University Of Burdwan) Pin -712104,India
}

\begin{abstract}
Computers have become indispensable in all domains, and the medical segment does not represent an exception. The need for accuracy and speed has led to a tight collaboration between machines and human beings. Maybe the future will allow the existence of a world where the human intervention won't be necessary, but for now, the best approach in the medical field is to create semiautomatic applications, in order to help the doctors with the diagnoses, with following the patients' evolution and managing them and with other medical activities. Our application is designed for automatic measurements of orthopedic parameters, and allows the possibility of human intervention in case the parameters have not been detected properly. The segment of the application is Hip Arthroplasty. And Wavelet transforms and other multi-scale analysis functions have been used for compact signal and image representations in de-noising, compression and feature detection processing problems for about twenty years. Numerous research works have proven that space-frequency and space-scale expansions with this family of analysis functions provided a very efficient framework for signal or image data. The wavelet transform itself offers great design flexibility. Basis selection, spatial-frequency tiling, and various wavelet threshold strategies can be optimized for best adaptation to a processing application, data characteristics and feature of interest. Fast implementation of wavelet transforms using a filter-bank framework enable real time processing capability. Instead of trying to replace standard image processing techniques, wavelet transforms offer an efficient representation of the signal, finely tuned to its intrinsic properties. By combining such representations with simple processing techniques in the transform domain, multi-scale analysis can accomplish remarkable performance and efficiency for many image processing problems. Multiscale analysis has been found particularly successful for image de-noising and enhancement problems given that a suitable separation of signal and noise can be achieved in the transform domain (i.e. after projection of an observation signal) based on their distinct localization and distribution in the spatial-frequency domain. With better correlation of significant features, wavelets were also proven to be very useful for detection \{jin_Mallat_1992a\} and matching applications \{jin_Strickland_1995\}.
\end{abstract}

Key-Words - Hip Arthroplasty, Canny Edge Detection, DICOM, Hough Transform, Radiographic Image

Processing De-noising, Segmentation

\section{Introduction}

Medical image processing is an area of increasing interest. It includes a wide range of methods and techniques, starting with the acquisition of images using specialized devices (for example, CT devices), image enhancement and analysis, to 3D model reconstruction from 2D images. Thus, the research in this field represents a point of interest for both doctors and engineers, in their attempt to improve medical techniques, with computer assistance, in order to obtain more accurate results in treating the patients. Among many research projects in this area of interest some of the most relevant are: The SCANIP [11] image processing software that provides a broad range of image visualization, processing and segmentation tools for medical purposes. This software has been created by Simpleware in Great Britain. The SCANIP programs ensure the conversion of 3D medical images into quality meshes. These meshes can be used in future processing in analysis programs, in fluid dynamics, CAD and the creation of Rapid Prototyping models. The sources for these programs come from MRIs, CTs or MicroCTs. The 3D-DOCTOR Project [12] that comes with an advanced 3d modeling software, with strong processing and measurement functions for MRI-s, CT-s, PET-s, and other types of medical images. The possible applications of this software are in the scientific and medical domain, but also in the image processing industrial field. The functioning principle of this software is based on edge detection techniques using 3D image segmentation functions and on the construction of 3D surfaces and volumes, that are afterwards visualized and measured for the purpose of a quantitative and qualitative analysis. - The Hip-OpCT software [13] that allows importing CT images in DICOM format. Once imported, the CT dataset is visualized through several modalities from which the doctors can plan the size and the position of the prosthesis. Wavelets have been widely used in signal and image processing for the past 20 years. Although a milestone paper by Grossmann and Morlet \{jin_Grossman_1984\} was considered as the beginning point of modern wavelet analysis, similar ideas and theoretical bases can be found back in early $20{ }^{\text {th }}$ century \{jin_Haar_1910\}. Following 
two important papers in late 1980s by S. Mallat \{jin_Mallat_1989\} and I. Daubechies \{jin_Daubechies_1988\}, more than 9,000 journal papers and 200 books related to wavelets have been published \{jin_Unser_2003a\}.

Wavelets were first introduced to medical imaging research in 1991 in a journal paper describing the application of wavelet transforms for noise reduction in MRI images. \{jin_Weaver_1991\}. Ever since, wavelet transforms have been successfully applied to many topics including tomographic reconstruction, image compression, noised reduction, image enhancement, texture analysis/segmentation and multi-scale registration. Two review papers in 1996 \{jin_Unser_1996\} and 2000 \{jin_Laine_2000\} provide a summary and overview of research works related to wavelets in medical image processing from the past few years. Many related works can also be found in the book edited by A. Aldroubi and M. Unser \{jin_Aldroubi_1996\}. More currently, a special issue of IEEE Transactions on Medical Imaging \{jin_Unser_2003a\} provides a large collection of most recent research works using wavelets in medical image processing. The purpose of this chapter is to summarize the usefulness of wavelets in various problems of medical imaging. The chapter is organized as follows. Section 2 overviews the theoretical fundamentals of wavelet theory and related multi-scale representations. As an example, the implementation of an over-complete dyadic wavelet transform will be illustrated. Section 3 includes a general introduction of image de-noising and enhancement techniques using wavelet analysis. Section 4 and 5 will summarize the basic principles and research works in literature for wavelet analysis applied to image segmentation and registration.

\section{Arthroplasty- General Presentation}

Arthroplasty [10] represents a surgical procedure in which the arthritic or dysfunctional joint surface is replaced with prosthesis or by remodeling or realigning the joint. The important joint for this article is the one located at hip area. This is the reason why the article details the parameters that belong to the thigh-bone and the pelvis. Fig.1 presents the most important parameters in Hip Arthroplasty, extracted from an anterior-posterior radiography. Fig. 2 presents the parameters extracted from an anterior-lateral radiography representing the hip after the insertion of the prosthesis. For the scope of this article, three areas of the thighboneare analyzed: the femoral head (the nearest part to the pelvis), the femoral neck, and the femoral shaft or body (the longest part of the thigh-bone).



Fig.1. Parameters important in Hip Replacement, extracted from an anterior-posterior radiography

The parameters of interest for the Hip Replacement [7] are listed below:

1) the superior margin of the acetabulum (the superior point in which the thigh-bone meetsthe pelvis). 2) the inferior margin of the acetabulum (the inferior point in which the thigh-bone (thefemoral head) meets the pelvis).3) the femoral head axis or the acetabulum axis (the line determined by the two points that represent the superior margin and the inferior margin of the acetabulum). 4) the angle created by the acetabulum axis with the vertical line. This angle has to be the same for both of the femoral bones. 5) the lesser trochanter (located in the upper left part of the femoral body). 6) the greater trochanter (located in the upper right part of the femoral body). 7) the tangent to the superior cortical of the femoral neck 8) the tangent to the inferior cortical of the femoral neck 9) the femoral neck axis (the axis of the cylinder determined by the tangents 7 and 8) 10) the femoral body axis or the diaphyseal axis (the axis of the cylinder that approximates the femoral body) 11) the most important parameter, extracted from the $\mathrm{x}$-ray before the surgical intervention is represented by the cervicodiaphyseal angle (the angle determined by the neck axis and the diaphyseal axis). Depending on the value of this angle, it can be determined whether the patient needs or not a prosthesis. If the angle has values between 125 and 135 degrees, the thigh-bone is considered to be in normal ranges. 12) the right ischiadic tuberosity (the lowest right part of the pelvic bone). 13) the left ischiadic tuberosity (the lowest left part of the pelvic bone).14) the ischiadic line or the horizontal reference line (the line determined by the two ischiadic tuberosities). 15) the vertical reference line, that is perpendicular on the ischiadic line, 
in its middle. 16) the line starting from the center of the lesser left trochanter, parallel to the ischiadic line 17) the line starting from the center of the right lesser trochanter, parallel to the ischiadic line; the distance between lines 16 and 17 represents the vertical distance between the two thigh-bones. If this distance is greater than a chosen threshold, there is an indication of a difference between the lengths of the two femoral bones that has to be resolved surgically (in most cases). After inserting the prosthesis, some new parameters must be taken into consideration: 18) the diaphyseal axis of the femoral bone (the same as parameter 10). 19) the diaphyseal axis of the prosthesis or the axis of the prosthesis' body (the axis of the cylinder that approximates the prosthesis' body). 20) The deviation of the prosthesis (the angle determined by lines 18 and 19). This parameter will be computed in several radiographic images, following the evolution of the same patient. The important parameters extracted from an anteriorlateral radiography, after inserting the prosthesis, are shown in Fig.2:

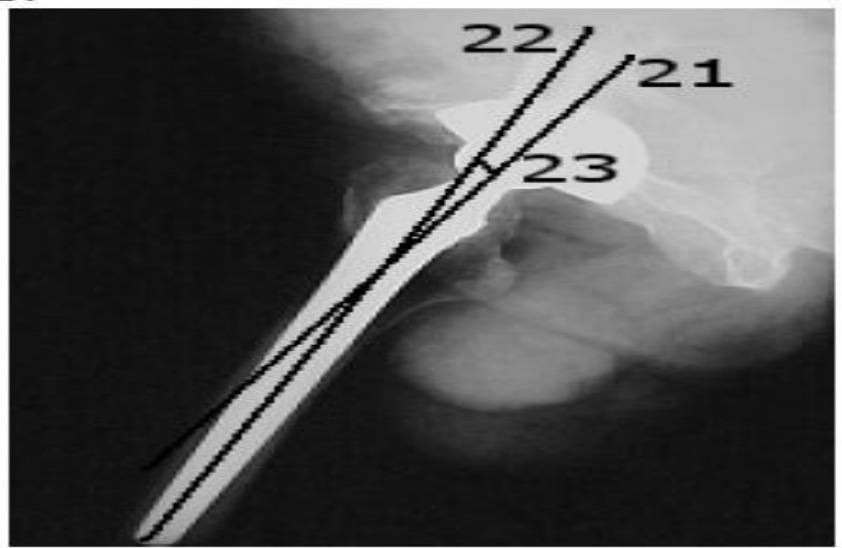

Fig.2. Parameters important in Hip Replacement, extracted from an anterior-lateral radiography, after the insertion of the prosthesis

21) The axis of the prosthesis' neck 22) The axis of the prosthesis' body 23) The anteversion angle (the angle determined by the axes 21 and 22). If this angle has its value situated between 5 and 10 degrees, it's considered to be in normal ranges.

\section{The DICOM Standard}

In order to manage and interpret the $\mathrm{x}$-rays data in a simple and organized manner, a standard for the $\mathrm{x}$ ray files is needed. This is the reason why the most popular standard for medical images was chosen: DICOM [14]. The Digital Imaging and Communications in Medicine (DICOM) standard is a detailed specification of the coding and transfer of medical images and their associated information.

\section{Representing Data in the DICOM Format}

The clinical data are represented in a variety of formats: the distances are measured in millimeters, the time in seconds, etc. The PS 3.5 part of the standard, entitled Data Structure and Their Encoding, defines 27 types of standard data, known as "value representations" (VR), that include all types of data that can appear in the medical domain. Any information encoded in a DICOM file has to belong to one of these predefined types.

Some of the most important standard data are: Person Name (PN), Date Time (DT), and Age String (AS). A DICOM file has the following structure: - A preamble of 128 bytes - A prefix (4 bytes) for retaining the letters 'D', 'I', 'C', 'M' (the signature of a DICOM file) - A data set that contains information like: patient's name, image type, image dimension, etc. - Pixels that form the image (or the images) contained in the file.

\section{Extracting Data from DICOM Files}

Extracting data from a DICOM file can be made using the tags defined in the DICOM dictionary. Every tag is searched in the file, and, if found, is interpreted. The steps in extracting the information are: Checking the existence of the characters 'D', 'I', 'C', 'M' - Determining of the VR type - Setting the order of the bytes (Big Endian or Little Endian) - Searching for a tag in the DICOM file, corresponding to the order of bytes and the VR type - Extracting the values corresponding to that tag Some characteristics of the DICOM files, important when extracting data, are: - the number of images contained in the DICOM file - the number of bits per pixel: $8,12,16,24$ - the compression - the photometric interpretation: shades of gray or color images In case of images without compression, the extraction of the images is made pixel by pixel, according to the number of bits per pixel. For images with compression, a decompression step should be previously performed. 


\section{The Structure of the CR DICOM files}

Computer radiographic images (CR) stored in DICOM files are accompanied by general identification elements and some specific information. For example, the Patient module contains: the name of the patient, the patient's ID, the patient's date of birth, etc. Another module, specific for CR, CR Series, contains information about the examined body part, the view position, etc. Our application extracts from every module the important elements for managing and interpreting the patient's data.

\section{Wavelet Transform And Multi-Scale Analysis}

One of the most fundamental problems in signal processing is to find a suitable representation of the data that will facilitate an analysis procedure. One way to achieve this goal is to use transformation, or decomposition of the signal on a set of basis functions prior to processing in the transform domain. Transform theory has played a key role in image processing for a number of years, and it continues to be a topic of interest in theoretical as well as applied work in this field. Image transforms are used widely in many image processing fields, including image enhancement, restoration, encoding, and description \{jin_Jain_1989\}. Historically, the Fourier transform has dominated linear time-invariant signal processing. The associated basis functions are

complex sinusoidal waves $e^{i \omega t}$ that correspond to the eigenvectors of a linear time-invariant operator. A signal $f(t)$ defined in the temporal domain and its Fourier transform $\hat{f}(\omega)$ defined in the frequency domain, have the following relationships \{jin_Jain_1989; jin_Papoulis_1987\}:

$$
\begin{aligned}
& \hat{f}(\omega)=\int_{-\infty}^{+\infty} f(t) e^{-i \omega t} d t, \\
& f(t)=\frac{1}{2 \pi} \int_{-\infty}^{+\infty} \hat{f}(\omega) e^{i \omega t} d \omega .
\end{aligned}
$$

Fourier transform characterizes a signal $f(t)$ via its frequency components. Since the support of the bases function $e^{i \omega t}$ covers the whole temporal domain (i.e infinite support),<smiles>[13CH3]</smiles>
depends on the values of $f(t)$ for all times. This makes the Fourier transform a global transform that cannot analyze local or transient properties of the original signal $f(t)$ In order to capture frequency evolution of a non-static signal, the basis functions should have compact support in both time and frequency domain. To achieve this goal, a windowed Fourier transform (WFT) was first introduced with the use of a window function $w(t)$ into the Fourier transform \{jin_Mallat_1998\}:

$$
S f(\omega, t)=\int_{-\infty}^{+\infty} f(\tau) w(t-\tau) e^{-i \omega \tau} d \tau \text {. }
$$

The energy of the basis function $g_{\tau, \xi}(t)=w(t-\tau) e^{-i \xi t}$ is concentrated in the neighborhood of time $\tau$ over an interval of size $\sigma_{t}$ measured by the standard deviation of $|g|^{2}$ Its Fourier transform is $\hat{g}_{\tau, \xi}(\omega)=\hat{w}(\omega-\xi) e^{-i \tau(\omega-\xi)}$

$\xi$ over an interval of size $\sigma_{\omega}$ In a time-frequency plane $(t, \omega)$ the energy spread of what is called the atom $g_{\tau, \xi}(t)$ is represented by the Heisenberg rectangle with time width $\sigma_{t}$ and frequency width $\sigma_{\omega} \cdot$ The

uncertainty principle states that the energy spread of a function and its Fourier transform cannot be simultaneously arbitrarily small, verifying:

$$
\sigma_{t} \sigma_{\omega} \geq \frac{1}{2}
$$

Shape and size of Heisenberg rectangles of a windowed Fourier transform therefore determine the spatial and frequency resolution offered by such transform. Examples of spatial-frequency tiling with Heisenberg rectangles are shown in Fig 3. Notice that for a windowed Fourier transform, the shape of the time-frequency boxes are 
identical across the whole time-frequency plane, which means that the analysis resolution of a windowed Fourier transform remains the same across all frequency and spatial locations.

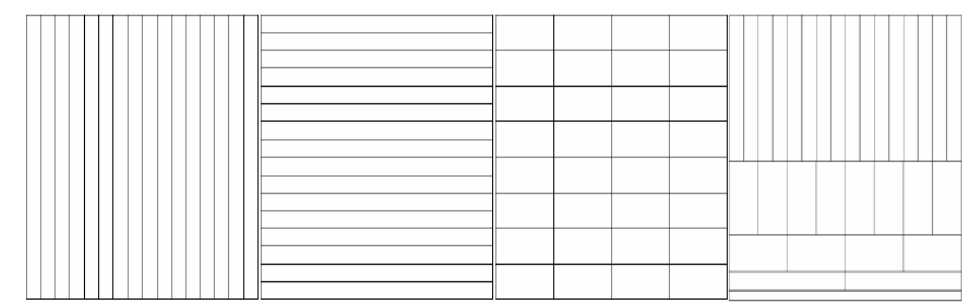

(b)

(d)

Fig .3. Example of spatial-frequency tiling of various transformations. $x$-axis: spatial resolution. $y$-axis: frequency resolution. (a) discrete sampling (no frequency localization ). (b) Fourier transform (no temporal localization). (c) windowed Fourier transform (constant Heisenberg boxes). (d) wavelet transform (variable Heisenberg boxes).

To analyze transient signal structures of various supports and amplitudes in time, it is necessary to use time-frequency atoms with different support sizes for different temporal locations. For example, in the case of high frequency structures, which vary rapidly in time, we need higher temporal resolution to accurately trace the trajectory of the changes; on the other hand, for lower frequency, we will need a relatively higher absolute frequency resolution to give a better measurement on the value of frequency. We will show in the next section that wavelet transform provide a natural representation which satisfies these requirements, as illustrated in Fig. 3 (d).

\section{Noise Reduction and Image Enhancement Using Wavelet Transforms}

De-noising can be viewed as an estimation problem trying to recover a true signal component $\mathrm{X}$ from an observation $\mathrm{Y}$ where the signal component has been degraded by a noise component $\mathrm{N}$ :

$$
Y=X+N \text {. }
$$

The estimation is computed with a thresholding estimator in an orthonormal basis

$$
\mathbf{B}=\left\{g_{m}\right\}_{0 \leq m<N}
$$

\{jin_Donoho_1994b\}:

$$
\hat{X}=\sum_{m=0}^{N-1} \rho_{m}\left(\left\langle X, g_{m}\right\rangle\right) g_{m}
$$

where $\rho_{m}$ is a thresholding function that aims at eliminating noise components (via attenuating of decreasing some coefficient sets) in the transform domain while preserving the true signal coefficients. If the

function $\mathcal{P}_{m}$ is modified to rather preserve or increase coefficient values in the transform domain, it is possible to enhance some features of interest in the true signal component with the framework of Equation (6). Fig. 4 illustrates a multi-scale enhancement and de-noising framework using wavelet transforms. An overcomplete dyadic wavelet transform using bi-orthogonal basis is used. Notice that since the DC-cap contains the overall energy distribution, it is usually kept untouched during the procedure. As shown in this figure, thresholding and enhancement functions can be implemented independently from the wavelet filters and easily incorporated into the filter bank framework.

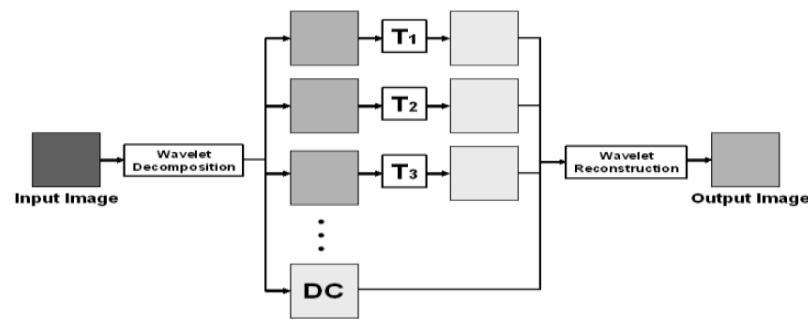

Fig .4. A Multi-scale framework of de-noising and enhancement using discrete dyadic wavelet transform. A

\section{Thresholding operators for de-noising} three level decomposition was shown.

As a general rule, wavelet coefficients with larger magnitude are correlated with salient features in the image data. In that context, de-noising can be achieved by applying a thresholding operator to the wavelet 
coefficients (in the transform domain) followed by reconstruction of the signal to the original image (spatial) domain.

Typical threshold operators for de-noising include hard thresholding

$$
\rho_{T}(x)=\left\{\begin{array}{ll}
x, \text { if } & |x|>T \\
0, \text { if } & |x| \leq T
\end{array} ;\right.
$$

soft thresholding (wavelet shrinkage) \{jin_Donoho_1995b\}:

$$
\rho_{T}(x)=\left\{\begin{array}{clc}
x-T, & \text { if } & x \geq T \\
x+T, & \text { if } & x \leq-T \\
0, & \text { if } & |x|<T
\end{array}\right.
$$

and affine(firm) thresholding \{jin_Gao_1997\}:

$$
\rho_{T}(x)=\left\{\begin{array}{clc}
x, & \text { if } & |x| \geq T \\
2 x+T, & \text { if } & -T \leq x \leq-T / 2 \\
2 x-T, & \text { if } & T / 2 \leq x \leq T \\
0, & \text { if } & |x|<T
\end{array} .\right.
$$

The shapes of these thresholding operators are illustrated in Fig 5.

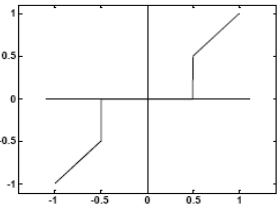

(a)

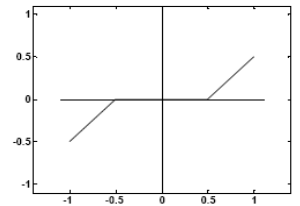

(b)

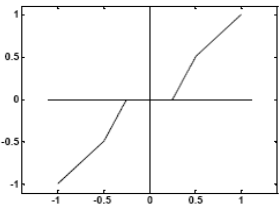

(c)

Fig .5. Example of thresholding functions, assuming that the input data was normalized to the range of $[-1,1]$. (a) Hard thresholding. (b) Soft thresholding. (c) Affine thresholding. The threshold level was set to T=0.5;

\section{Image Segmentation Using Wavelets}

\subsection{Multi-scale Texture Classification and Segmentation}

Texture is an important characteristic for analyzing many types of images, including natural scenes and medical images. With the unique property of spatial-frequency localization, wavelet functions provide an ideal representation for texture analysis. Experimental evidence on human and mammalian vision support the notion of spatial-frequency analysis that maximizes a simultaneous localization of energy in both spatial and frequency domain \{jin_Beck_1987; jin_Julez_1981; jin_Watson_1983\}. These psychophysical and physiological findings lead to several research works on texture-based segmentation methods based on multi-scale analysis. Gabor transform, as suggested by the uncertainty principle, provides an optimal joint resolution in the space-frequency domain. Many early works utilized Gabor transforms for texture characteristics. In \{jin_Daugman_1988\} an example is given on the use of Gabor coefficient spectral signatures \{jin_Daugman_1985\} to separate distinct textural regions characterized by different orientations and predominant anisotropic texture moments. Porat and Zeevi proposed in \{jin_Porat_1989\} six features derived from Gabor coefficients to characterize a local texture component in an image: the dominant localized frequency; the second moment (variance) of the localized frequency; center of gravity; variance of local orientation; local mean intensity; and variance of the intensity level. A simple minimum-distance classifier was used to classify individual textured regions within a single image using these features. Many wavelet-based texture segmentation methods had been investigated thereafter. Most of these methods follow a three-step procedure: multi-scale expansion, feature characterization, and classification. As such, they are usually different from each other from these aspects. Various multi-scale representations have been used for texture analysis. Unser \{jin_Unser_1995a\} used a redundant wavelet frame.

Laine and Fan \{jin_Laine_1993\} investigated a wavelet packets representation and extended their research to a redundant wavelet packets frame with Lemarié-Battle filters in \{jin_Laine_1996a\}. Modulated wavelets were used in \{jin_Hsin_1998\} for better orientation adaptivity. To further extend the flexibility of the spatial-frequency analysis, a multi-wavelet packets, combining multiple wavelet basis functions at different expansion levels was used in \{jin_Wang_2002\}. An M-band wavelet expansion, which differs from a dyadic wavelet transform in the fact that each expansion level contains $M$ channels of analysis was used in \{jin_Acharyya_2002\} to improve orientation selectivity. Quality and accuracy of segmentation ultimately depends on the selection of the characterizing features. A simple feature selection can use the amplitude of the wavelet coefficients \{jin_Hsin_1998\}. Many multi-scale texture segmentation methods construct the feature 
vector from various local statistics of the wavelet coefficients, such as its local variance \{jin_Unser_1995a; jin_Wang_2001\}, moments \{jin_Etemad_1997\} or energy signature \{jin_Acharyya_2002; jin_Laine_1993; jin_Porter_1996\}. Wavelet extrema density (WED), defined as the number of extrema of wavelet coefficients per unit area, was used in \{jin_Wang_2002\}. In \{jin_Laine_1996a \}, a 1-D envelope detection was first applied to the wavelet packets coefficients according to their orientation, and a feature vector was constructed as the collection of envelope values for each spatial-frequency component. More sophisticated statistical analysis involving Bayesian analysis and Markov random fields were also used to estimate local and long-range

correlations $\left\{\right.$ jin_Choi_2001; jin_Zhang_1998\}. For other examples of multi-scale textural features, $\mathcal{X}^{2}$ test and histogram testing were used in $\{$ jin_Li_2000\}, "Roughness" based on fractal dimension measurement was used in \{jin_Charalampidis_2002\}. Texture-based segmentation is usually achieved by texture classification. Classic classifiers such as the minimum distance classifier \{jin_Porat_1989\}, are easier to implement when the dimension of the feature vector is small and the groups of samples are well segregated. The most popular classification procedures reported in the literature are the K-mean classifier \{jin_Acharyya_2002; jin_Charalampidis_2002; jin_Hsin_1998; jin_Laine_1996a; jin_Porter_1996; jin_Unser_1995a; jin_Wang_2001\} and the neural networks classifiers \{jin_Daugman_1988; jin_Etemad_1997; jin_Laine_1993; jin_Zhang_1998\}.
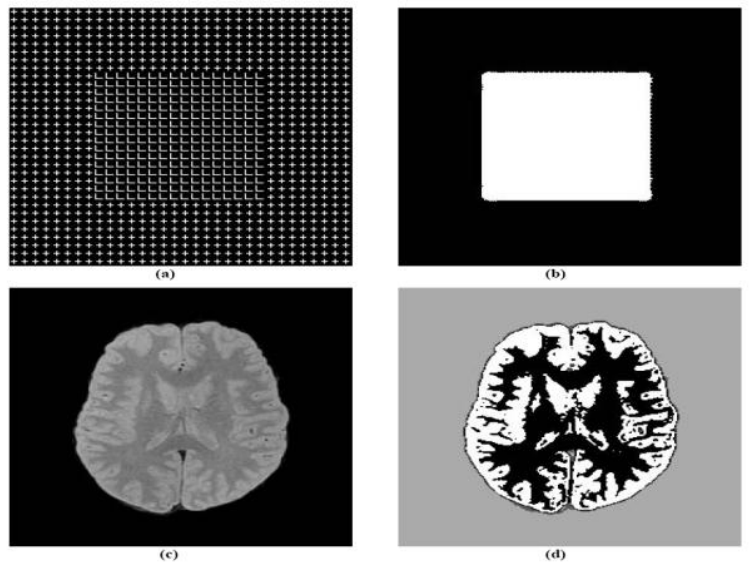

Fig .6. Sample results using multi-scale texture segmentation. (a) Synthetic texture image. (b): segmentation result for image (a) with a 2-class labeling. (c) MRI T1 image of a human brain. (d) segmentation result for image (c) with a 4-class labeling.

As an example, we illustrate in Fig. 6 a texture-based segmentation method on both a synthetic texture image and a medical image from a brain MRI data set. The algorithm used for this example from \{jin_Laine_1996a\} uses the combination of wavelet packets frame with Lemarié-Battle filters, multi-scale envelope features, and a K-mean classifier.

\section{Wavelet Edge Detection and Segmentation}

Edge detection plays important role in image segmentation. In many cases, boundary delineation is the ultimate goal for an image segmentation and a good edge detector itself can then fulfill the requirement of segmentation. On the other hand, many segmentation techniques require an estimation of object edges for their intialization. For example, with standard gradient-based deformable models, an edge map is used to determine where the deforming interface must stop. In this case, the final result of the segmentation method depends heavily on the accuracy and completeness of the initial edge map. Although many research works have made some efforts to eliminate this type of inter-dependency by introducing non-edge constraints \{jin_Chan_2001; jin_Yezzi_1999\}, it is necessary and equally important to improve the edge estimation process itself. As pointed out by the pioneer work of Mallat and Zhong \{jin_Mallat_1992b\}, first or second derivative based wavelet functions can be used for multi-scale edge detection. Most multi-scale edge detectors smooth the input signal at various scales and detect sharp variation locations (edges) from their first or second derivatives. Edge locations are related to the extrema of the first derivative of the signal and the zero crossings of the second derivative of the signal. In \{jin_Mallat_1992b\}, it was also pointed out that first derivative wavelet functions are more appropriate for edge detection since the magnitude of wavelet modulus represents the relative "strength" of the edges, and therefore enable to differentiate meaningful edges from small fluctuations caused by noise.

Using the first derivative of a smooth function $\theta(x, y)$ as the mother wavelet of a multi scale expansion results in a representation where the two components of wavelet coefficients at a certain scale s are 
related to the gradient vector of the input image $f(x, y)$ smoothed by a dilated version of $\theta(x, y)$ at scale s.

$$
\left(\begin{array}{l}
W_{s}^{1} f(x, y) \\
W_{s}^{2} f(x, y)
\end{array}\right)=s \vec{\nabla}\left(f * \theta_{s}\right)(x, y) .
$$

The direction of the gradient vector at a point $(\mathrm{x}, \mathrm{y})$ indicates the direction in the image plane along which the directional derivative of $\mathrm{f}(\mathrm{x}, \mathrm{y})$ has the largest absolute value. Edge points (local maxima) can be detected as points $00($,$) xysuch that the modulus of the gradient vector is maximum in the direction towards$ which the gradient vector points in the image plane. Such computation is closely related to a Canny edge detector \{jin_Canny_1986\}. Extension to higher dimension is quite straightforward. Fig. 7 provides an example of a multi-scale edge detection method based on a first derivative wavelet function. To further improve the robustness of such multi-scale edge detector, Mallat and Zhong \{jin_Mallat_1992b\} also investigated the relations between singularity (Lipschitz regularity) and the propagation of multi-scale edges across wavelet scales. In \{jin_Aydin_1996\}, the dyadic expansion was extended to an M-band expansion to increase directional selectivity. Also, continuous scale representation was used for better adaptation to object sizes \{jin_Laine_1997\}. Continuity constraints were applied to fully recover a reliable boundary delineation from 2D and 3D cardiac ultrasound in \{jin_Laine_1996b\} and \{jin_Koren_1994\}. In \{jin_Dima_2002\}, both cross-scale edge correlations and spatial continuity were investigated to improve the edge detection in the presence of noise.

Wilson et al. in \{jin_Wilson_1992\} also suggested that a multi-resolution Markov model can be used to track boundary curves of objects from a multi-scale expansion using a generalized wavelet transform.
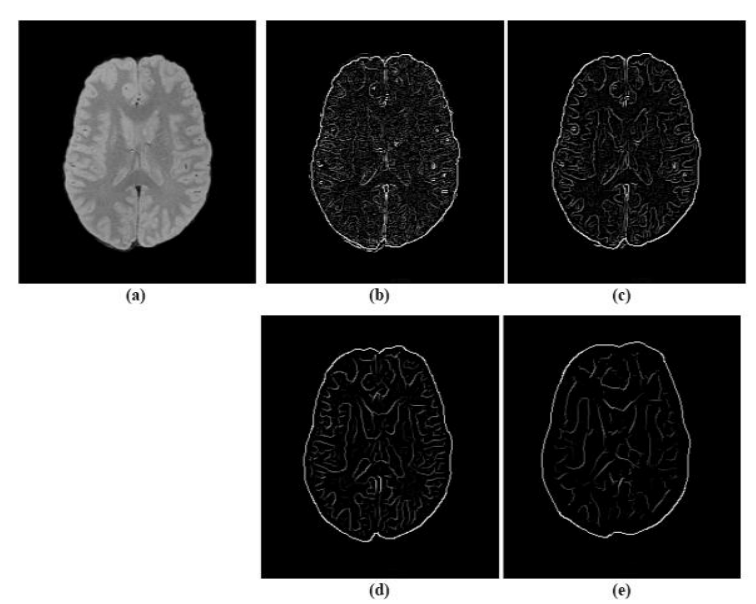

Fig.7. Example of a multi-scale edge detection method finding local maxima of wavelet modulus, with a firstderivative wavelet function. (a) Input image, (b) (e): Multi-scale edge map at expansion scale 1 to 4.

Given their robustness and natural representation as boundary information within a multi-resolution representation, multi-scale edges have been used in deformable model methods to provide a more reliable constraint on the model deformation \{jin_de Rivaz_2000; jin_Sun_2003; jin_Wu_2000; jin_Yoshida_1997\}, as an alternative to traditional gradient based edge map. In \{jin_Neves_2003\}, it was used as a pre-segmentation step in order to find the markers that are used by watershed transform.

\section{Radiographic Image Processing}

As in any image analysis application, the first step is a preprocessing step, needed to improve the image by noise removal, contrast improvement, edge enhancement and others [4]. In our application, this step is followed by a contour extraction step, which helps in the arthroplasty parameters' extraction.

\section{Image Enhancement}

One reason why the automatic interpretation of radiographic images doesn't give accurate results is the fact that the radiographic images are blurred. This is why enhancing images before applying contour detection algorithms is a step that should not be omitted. In the case of our application, the radiographic images are enhanced by noise removal, edge enhancement and contrast improvement. We will detail each method in the following subsections. 


\section{Noise Removal}

A series of methods have been tested in order to choose the best way for noise removal.The first approach was that of using Gaussian filtering, that led to good results. Another interesting approach would be Impulsive Noise removal [15]. The classic linear unsharp masking is an important scheme in feature based image enhancement category, in which a high pass filter scaled version of the original image is added to itself, in order to give more emphasis on the high frequency components. There is a very big possibility that the noise will be amplified along with the detail features of the image. Thus, the main objective of this approach is to separate the noisy and the noise-free images based on a certain threshold. The creators of the method propose the use of a CV (coefficient of variance) based adaptive threshold technique for impulsive noise detection. The main idea is to train a functional link artificial neural network (FLANN) [16] with a single statistical parameter $\mathrm{CV}$ as input. The third approach meant for image enhancement is Image Noise removal using Graph Theory Concepts [17]. This method first creates an image graph, based on the original image. There are many possible mappings, but the most intuitive approach is to map each pixel in the image onto a vertex of the graph. This graph will be four-way connected, so that each vertex corresponds to a pixel, and each edge has an associated weight that corresponds to the distance between the color levels of the adjacent vertices. This technique has been tested for impulse noise and for random noise, leading to the conclusion that the proposed method displays good edge and detail preservation, while suppressing impulse and random noise. The chosen method for noise removal was Gaussian Filtering, because of the need for speed in this preprocessing phase.

\section{Edge Enhancement}

The first method used for edge enhancement was Adaptive Smoothing. This represents a filter that enhances edges, being applied like a matrix filter. The main difference between adaptive smoothing and matrix filter is the fact that the first does not apply weights over the pixel's neighborhood in order to compute its new intensity or color, but uses a gradient. The output obtained by using this gradient consists of both positive and negative intensities, but also emphasized high frequency details. Another method for edge enhancement was the curvelet transform [18]. Curvelet coefficient can be modified in order to enhance edges in an image by $\mathrm{y}(\mathrm{x})$ (Velde function). We have chosen the first method because it was faster.

\section{Contrast Improvement}

The method tried for contrast improvement was histogram equalization that usually increases the global contrast of images, especially when the usable data of images is represented by close contrast values. Peaks in the image histogram (that indicate commonly used grey levels) are widened, while valleys are compressed.

\section{Contour Extraction}

Most of the contour extraction algorithms which are based on edge detection follow these steps:

- detecting the edge pixels (pixels where the intensity changes abruptly) - eliminating the edge pixels which are not also contour pixels - connecting the contour pixels using local methods (based on the pixels' relations to their neighbor pixels) or global methods (based on global information, for example the shape of a bone, in a computer radiography). After trying a series of methods, the Canny algorithm [5] has been chosen in order to extract the contour lines, because this produced the best results. The Canny algorithm will be briefly described in the following lines. The Canny edge detection algorithm is a very well known algorithm and is considered by many the optimal edge detector. The algorithm is structured into 6 steps: 1) filter out any noise in the original image with the Gaussian filter 2) apply the Sobel operator on the resulting image, estimating the gradient in the horizontal direction $(\mathrm{Gx})$ and in the vertical direction (Gy). The magnitude, or the edge strength is approximated by the sum between Gx and Gy

3) find the edge direction, as the arctangent of Gy/Gx. 4) Once the direction is known, relate the edge direction to a discretized direction (all the angles between 67.5 and 112.5 will be considered to be of 90 degrees, all angles between 112.5 and 157.5 are set to 135 degrees, etc). Fig. 8 shows the possible discretized edge directions, previously determined in step 3 . 


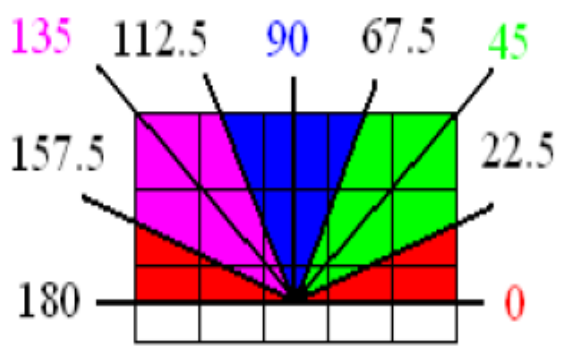

Fig. 8. Gradient direction

5) Non-maximum suppression (trace along the edge in the edge direction and suppress any pixel that is not considered to be an edge - that is not a local maximum) 6) Hysteresis for eliminating streaking, using two thresholds, T1 (high) and T2 (low). Any pixel with a value greater than T1 is considered to be an edge pixel.

After applying the first threshold, any pixels connected to the edge pixels that have a value greater than $\mathrm{T} 2$ will also be selected as edge pixels. Usually $\mathrm{T} 1=2 * \mathrm{~T} 2$. The result of applying the Canny detector is a binary image (Fig. 9), where white pixels represent contour pixels. Having the contour lines of the bones, the next step in our application is the extraction of the important parameters in hip arthroplasty (automatic and semiautomatic extraction). We can observe in Fig. 4 that the contour is disconnected in some parts of the radiography, and that it cannot be reconstructed with accuracy. That is why we propose to search for some salient parameters (for example the lines representing the contour of the femoral body) that can be identified without the previous reconstruction of the entire pelvic and femoral contour. In the following section we will present the methods used after preprocessing the image (with noise removal, edge enhancement, contrast improvement and Canny edge detection), in order to extract the parameters important in arthroplasty.



Fig.9. The image after applying the Canny Edge Detector

\section{Conclusions}

The research in the field of medical image analysis is a continuous challenge. The need to discover new image analysis algorithms and new automatic learning techniques that would help in computer assisted diagnosis is and will be a topic of interest for researchers. The results of our research, presented in this paper,prove that solutions do exist. Although not all the arthroplasty parameters determined automatically were $100 \%$ accurate, the application proved to be very useful to doctors. The fact that the application allows patient's data saving management, during a long period of time after the hip replacement procedure is another plus. This application can be used for a single hospital, or for an entire national/international network of hospitals, integrating other applications of diagnosis or of assisting doctors in planning certain surgeries and following the patients' evolution after the surgeries.

The versatility of these multi-scale transforms make them a suitable tool for several applications in signal and image processing that can benefit from the following advantages: 1. A wavelet transform decomposes a signal to a hierarchy of sub-bands with sequential decrease in resolution. Such expansions are especially useful when a multi-resolution representation is needed. Some image segmentation and registration techniques can benefit from a "coarse to fine" paradigm based on a multi-resolution framework. 2. A signal can be analyzed with a multi-resolution framework into a spatial-frequency representation. By carefully selecting the wavelet function and the space-frequency plane tiling of the transform, distinct components from a noisy observation signal can be easily separated based on their spatial-frequency characteristics. 3. Many important features from an image data can be characterized more efficiently in the spatial-frequency domain. Such feature 
characterization was shown to be extremely useful in many applications including registration and data compression. We summarized in this chapter some important applications in medical image processing using wavelet transforms. Noise reduction and enhancement can be easily implemented by combining some very simple linear thresholding techniques with wavelet expansion. Efficient de-noising and enhancement improve image quality for further analysis including segmentation and registration. Feature characteristics in wavelet domain were proven to be potentially more efficient and reliable when compared to spatial analysis only, and therefore provided more effective segmentation and registration algorithms. We point out that many other important applications of multi-resolution wavelet transforms, that were beyond the scope of this book, have not been covered in this chapter, especially image compression, which is considered as one of the greatest achievement of wavelet transform in recent years \{jin_Unser_2003b $\}$. Other important applications include tomographic image reconstruction, analysis of functional MRI images (fMRI), and data encoding for MRI acquisition. Despite the great success of multi-resolutions wavelet transform in medical imaging applications for the past twenty years, it continues to be a very active area of research. We list a few resources below that are of interest to readers willing to get more knowledgeable in research and applications in this area.

\section{References}

[1] Image Analysis by local 2-D Spectral Signatures., Journal of Optical Society of American, A, Vol. 2, pp. 74 , 1985. lbibitem\{jin_Daugman_1988\} Daugman.

[2] Adapting to Unknown Smoothness via Wavelet Shrinkage., Journal of American Statistics Association, Vol. 90, No. 432, pp. 1200$1224,1995 \mathrm{c}$.

[3] Bankman I, Handbook of Medical Image Processing and Analysis, Academic Press, 2000.

[4] Feng D D, Biomedical Information Technology, Elsevier, 2008.

[5] Chen Y, Ee X, Leow K W, Howe T S, Automatic Extraction of Femur Contours from Hip X rayimages, 2000.

[6] Gonzales R C, Woods R E, Digital Image Processing, Prentice-Hall, 2002.

[7] Canny J F, A Computational Approach to Edge Detection, IEEE Trans. Pattern Analysis and Machine Intelligence, 1986.

[8] Campilho A, Kamel M, Image Analysis and Recognition, Springer. Raj K Sinha, Hip Replacement.

[10] Kennon R, Hip and Knee Surgery: A Patient's Guide to Hip Replacement, Hip Resurfacing, Knee Replacement, and Knee Arthroscopy Book Description.

[11] Botez P, Ortopedie, Bit Publishing House (Iasi), 2001.

[12] Morrey B F, Joint Replacement Arthtoplasty. SCANIP: Available http://www.simpleware.com/software/scanip/iptechd ata.php in 10.10.2009.

[14] 3D-DOCTOR: Available at http://www.ablesw.com/3d-doctor/index.html in 10.10.2009.

[15] Hip-OpCT: Available at http://www.hipop.it/hipopct.html in 20.10.2009.

[16] The official page of the DICOM standard. Availableat http://dicom.nema.org in 20.05.2009.

[17] Mohapatra S, Kumar Sa P, Majhi B, ImpulsiveNoise Removal Image Enhancement Technique, $6^{\text {th }}$ WSEAS International Conference on CIRCUITS, SYSTEMS, ELECTRONICS, CONTROL \& SIGNAL PROCESSING, Cairo, Egypt, 2007.

[18] Chen T, Wu HR, Adaptive impulse detection using center weighted median filters, IEEE Signal Process Lett, 2001.

[19] Georgakopoulos S, Andreadis A, Image Noise Removal Using Graph Theory Concepts, WSEAS Multiconference: Signal, Speech and Image Processing, Rethymno, Greece, 2003.

[20] Mohammad F, Al-Otum H M, Oraiqat M T, A Comparison of Image Enhancement using Curvelet Transform with Multiscale Gradient and Retinex Operators, WSEAS Multiconference: Signal, Speech and Image Processing, Izmir, Turkey, 2004.

[21] Djekoune O, Achour K, Halimi M, Kahlouche S, Incremental Hough Transform: An Improvement Algorithm for Digital Devices Implementatio.

[22] WSEAS Int. Conference on Electronics, Control \& Signal Processing and E-Activities, Singapore, 200 Ibibitem\{jin_Acharyya_2002\} Acharyya, M. and Kundu, M.

[23] Document Image Segmentation Using Wavelet Scale-Space Features., IEEE Trans. Circuits and Systems for Video Technology, Vol. 12, No. 12, pp. 1117-1127, 2002. Ibibitem\{jin_Aldroubi_1996\} Aldroubi, A. and Unser, M.

[24] Wavelets in Medicine and Biology. Boca Raton, FL: CRC, 1996. Vbibitem\{jin_Allen_1993\} Allen, R., Kamangar, F., and Stokely, E.

[25] Laplacian and Orthogonal Wavelet Pyramid Decompositions in Coarse-to-Fine Registration.,

IEEE Trans. Signal Processing, Vol. 41, No. 12, pp. 3536-3541, 1993. Ibibitem\{jin_Angelini_2001\} Angelini, E., Laine, A., Takuma, S., Holmes, J., and Homma, S.

[26] LV volume quantification via spatio-temporal analysis of real-time 3D echocardiography, IEEE Transactions on Medical Imaging, Vol. 20, pp. 457-469, 2001. Ibibitem\{jin_Antoniadis_2001\} Antoniadis, A. and Fan, J.

[27] Regularization of Wavelet Approximations., Journal of American Statistics Association, Vol. 96, No. 455, pp. 939-967, 2001. Jbibitem\{jin_Aydin_1996\} Aydin, T., Yemez, Y., Anarim, E., and Sankur, B.

[28] Multi-directional and Multi-scale Edge Detection via M-Band Wavelet Transform., IEEE Trans. Image Processing, Vol. 5, No. 9, pp. 1370-1377, 1996. Ibibitem\{jin_Babaud_1986\} Babaud, J., Witkin, A., Baudin, M., and Duba, R.

[29] Uniqueness of the Gaussian Kernel for Scale-space Filtering., IEEE Trans. Pattern Analysis and Machine Intelligence, Vol. 8, pp. 26-33, 1986. Ibibitem\{jin_Bastiaans_1981\} Bastiaans, M.

[30] A Sampling Theorem for the Complex Spectrogram and Gabor's Expansion of a Signal in Gaussian Elementary Signals., Optical Engineering, Vol. 20, No. 4, pp. 594-598, 1981. bibitem\{jin_Beck_1987\} Beck, J., Sutter, A., and Ivry, r.

[31] Spatial Freuqncy Channels and Perceptual Grouping in Texture Segregation., Computer Vision, Graphics, and Image Processing, Vol. 37, pp. 299-325, 1987. Ibibitem\{jin_Bello_1994\} Bello, M.

[32] A Combined Markov Random Field and Wave-Packet Transform-Based Approach for Image Segmentation., IEEE Trans. Image Processing, Vol. 3, No. 6, pp. 834-846, 1994.Jbibitem\{jin_Candes_1999a\} Candes, E. and Donoho, D.

[33] Curvelets - A Surprisingly Effective Nonadaptive Representation for Objects with Edges. in Curve and Surface Fitting: Saint-Malo 1999, Cohen, A., Rabut, C., and Schumaker, L., Eds. Nashville TN: Vanderbilt University Press, 1999a. lbibitem\{jin_Candes_1999b\} Candes, E. and Donoho, D. 
[34] Ridgelets: The Key to Higher-dimensional Intermittency?, Phil. Trans. R. Soc. Lond. A., Vol. 357, pp. 2495-2509, 1999b. lbibitem\{jin_Canny_1986\} Canny, J.

[35] A computational approach to edge detection, IEEE Transactions on Pattern Analysis and Machine Intelligence, Vol. 8, No. 6, pp. 679-698, 1986. Ibibitem\{jin_Chan_2001\} Chan, T. F. and Vese, L. A.

[36] Active Controus Without Edges, IEEE Transactions on Image Processing, Vol. 10, No. 2, pp. 266-277, 2001. Ibibitem\{jin_Chang_2000\} Chang, S., Yu, B., and Vetterli, M.

[37] Spatially adaptive wavelet thresholding with context modeling for image denoising, IEEE Transactions on Image Processing, Vol. 9, No. 9, pp. 1522-1531, 2000. Ibibitem\{jin_Charalampidis_2002\} Charalampidis, D. and Kasparis, T.

[38] Wavelet-based Rotational Invariant Roughness Features for Texture Classification and Segmentation., IEEE Trans. Image Processing, Vol. 11, No. 8, pp. 825-837, 2002. lbibitem\{jin_Choi_2001\} Choi, H. and Baraniuk, R.

[39] Multiscale Image Segmentation Using Wavelet-Domain Hidden Markov Models., IEEE Trans. Image Processing, Vol. 10, No. 9, pp. 1309-1321, 2001. Ibibitem\{jin_Coifman_1995a\} Coifman, R. and Donoho, D.

[40] Translation-invariant De-noising. in Wavelets and Statistics, Antoniadis, A. and Oppenheim, G., Eds. New York NY: SpringerVerlag, 1995a. Ibibitem\{jin_Coifman_1992\} Coifman, R. R., Meyer, Y., and Wickerhauser, M. V.

[41] Wavelet Analysis and signal processing in Wavelets and their applications, Ruskai, B., Ed. Boston: Jones and Barlett, pp. 153-178, 1992. Jbibitem\{jin_Coifman_1995b\} Coifman, R. R. and Woog, L. J.

[42] Adapted waveform analysis, wavelet packets, and local cosine libraries as a tool for image processing, Investigative and trial image processing, San Diego, California, Vol. 2567, 1995b. Ibibitem\{jin_Daubechies_1988\} Daubechies, I

[43] Orthonormal bases of compactly supported wavelets, Communications on Pure and Applied Mathematics, Vol. 41, No. 7, pp. 909996, 1988. Jbibitem\{jin_Daubechies_1992\}

Daubechies, I., Ten Lectures on Wavelets. Philadelphia, PA: Siam, 1992. Ibibitem\{jin_Daugman_1985\} Daugman, J.

[44] Complete Discrete 2-D Gabor Transforms by Neural Networks for Image Analysis and Compression.,

IEEE Trans. Acoustics, Speech, and Signal Processing, Ibibitem\{jin_de Rivaz_2000\} de Rivaz, P. and Kingsbury, N.

[45] Fast Segmentation Using Level Set Curves of Complex Wavelet Surfaces., IEEE International Conference on Image Processing, Vol. 3, pp. 29-32, 2000. Ibibitem\{jin_Dima_2002\} Dima, A., Scholz, M., and Obermayer, K.

[46] Automatic Segmentation and Skeletonization of Neurons From Confocal Microscopy Images Based on the 3-D Wavelet Transform., IEEE Trans. Image Processing, Vol. 11, No. 7, pp. 790-801, 2002. lbibitem\{jin_Dinov_2002\} Dinov, I., Mega, M., Thompson, P., Woods, R., Sumners, D., Sowell, E., and Toga, A.

[47] Quantitative Comparison and Analysis of Brain Image Registration Using Frequency-Adaptive Wavelet Shrinkage IEEE Trans. Information Technology in Biomedicine, Vol. 6, No. 1, pp. 73-85, 2002. Vbibitem\{jin_Donoho_1994a\} Donoho, D. and Johnstone, I.

[48] Ideal Spatial Adaptation via Wavelet Shrinkage., Biometrika, Vol. 81, pp. 425-455, 1994a. Ibibitem\{jin_Donoho_1995a\} Donoho, D.

[49] Nonlinear solution of linear inverse problems by wavelet-vaguelette decompositions, Journal of Applied and Computational Harmonic Analysis, Vol. 2, No. 2, pp. 101-126, 1995a. J Jibitem\{jin_Donoho_1995b\} Donoho, D.

[50] De-noising by Soft-thresholding., IEEE Trans. Information Theory, Vol. 41, No. 3, pp. 613-627, 1995b. Ibibitem\{jin_Donoho_1995c\} Donoho, D. and Johnstone, I. 